\title{
Pericardial Disease
}

Although echocardiography is considered the primary technique for evaluating the pericardium, magnetic resonance imaging (MRI) may be indicated in patients who have poor acoustic windows or who have complex pericardial disease. This unit presents the basic technique for evaluating nonneoplastic disease of the pericardium with optional contrast-enhanced sequences for evaluating suspected neoplastic disease. The parameters are based on experience on a Siemens 1.5 T Vision or Symphony and should be altered accordingly for different field strengths and manufacturers.

\section{IMAGING OF NONNEOPLASTIC PERICARDIUM}

To image the pericardium, cardiac imaging packages with gated echo-train fast (or turbo) spin-echo and segmented $k$-space cine gradient-echo images give better results in a significantly shorter examination time.

The core components of the imaging protocol are gated $T_{1}$-weighted and $T_{2}$-weighted turbo spin-echo images of the heart and pericardium, supplemented by a series of cine gradient echo images. The spin-echo images are used to provide high-resolution imaging of the pericardium and pericardial space and to help characterize effusions and masses. The electrocardiogram (ECG)-gated cine gradient-echo images generally provide views of a single two-dimensional (2-D) slice at multiple phases of the cardiac cycle. When viewed in the cine mode, these can be used to evaluate the motion of the heart dynamically. Cine MR images can help detect compression or diastolic collapse of the right side of the heart in cardiac tamponade and abnormal septal motion in patients with constrictive pericarditis. With fast breath-hold cine acquisitions, the total examination time for the Basic Protocol should be $<45$ min.

For patients with pericardial masses or possible neoplastic involvement of the pericardium, additional contrast-enhanced imaging of the pericardium can be performed by repeating $T_{1}$-weighted imaging following intravenous gadolinium chelate.

Table A11.1.1 lists the hardware necessary to perform MRI of the pericardium. Higher gradient strengths with imaging sequences that allow gated turbo spin-echo and segmented cine gradient-echo imaging are preferable.

NOTE: Be sure that technologists and nurses have immediate access to any emergency equipment that may be relevant to a given study, or that may be needed for a particular patient, such as a crash cart or oxygen. Also ensure only magnetic field compatible oxygen tanks are utilized if wall-source oxygen is unavailable.

Table A11.1.1 Equipment Parameters for Pericardial Imaging

\begin{tabular}{ll}
\hline Coil type & $\begin{array}{l}\text { Torso phased-array coil (or dedicated cardiac } \\
\text { coil, if available) }\end{array}$ \\
Gradient coil strength & $25-40 \mathrm{mT} / \mathrm{m}$ (or whatever the system permits) \\
Cardiac gating & Yes, preferably fiber-optic cables \\
Peripheral gating & No \\
Oxygen & Yes, 2 liters via nasal cannula for most patients \\
& (to ensure breath-holding $>15 \mathrm{sec}$ ) \\
Use of contrast agents & $\begin{array}{l}\text { Possible in patients with suspected neoplastic } \\
\text { disease (see Alternate Protocol) }\end{array}$ \\
\hline
\end{tabular}

Contributed by Vivian S. Lee

Current Protocols in Magnetic Resonance Imaging (2001) A11.1.1-A11.1.14

Copyright $\odot 2001$ by John Wiley \& Sons, Inc.
BASIC

PROTOCOL

Acquired Heart

Disease

A11.1.1 


\section{Set up equipment and patient}

1. Interview and screen the patient to ensure that he or she has no contraindications, such as cardiac pacemaker or defibrillator or other ferromagnetic materials near vital structures. Also, ensure that the patient does not have health conditions, including those related to cardiac or pericardial disease, that could require emergency equipment during the scanning procedure. Assess the need for the monitoring of blood pressure, pulse, and blood oxygenation during the examination.

Generally standard screening forms are used for all patients scanned in a magnetic resonance system.

The presence of any ferromagnetic metals may be a health hazard to the patient when he or she is inside the magnet and will also affect the imaging. If in doubt as to the exact composition of the items, it is best to exclude patients with any metal implants.

Patients may be accompanied into the magnet room by a friend or family member, who can sit in the room during the scan and comfort the patient as needed. This companion must be screened as well to ensure the absence of loose metal objects on the body or clothing.

2. If the procedure is a research protocol, have the patient sign any necessary consent form.

3. Have the patient remove all jewelry and change into a gown to eliminate any metal that might be found in clothing.

4. Inform the patient about what will occur during the procedure, what he or she will experience while in the magnet, and how to behave, including the following:

a. If earphones or headphones are used to protect the ears from the loud sounds produced by the gradients, the patient will be asked to wear these, but will be able to communicate with you at any time during the imaging.

b. The patient will be given a safety squeeze-bulb or similar equipment to request assistance at any time (demonstrate how this works).

c. For good results the patient should not talk, and should avoid or minimize swallowing or other movement, during each scan-i.e., as long as the banging sounds continue. Between scans, talking and swallowing are allowed in most cases, but should be avoided when comparative positional studies are being performed; the patient will be informed when this is the case.

d. Nevertheless, the patient may call out at any time if he or she feels it necessary.

5. Inform the patient of the need to hold his or her breath (preferably at end expiration) for $\sim 15$ to $20 \mathrm{sec}$ for each cine gradient-echo acquisition. Assess the need for supplementary oxygen to improve breath-holding capacity, and if necessary, administer 2 liters oxygen via nasal cannula. Advise the patient of the importance of not moving during the acquisition periods and of not taking deep breaths during the non-breath-hold acquisitions.

6. When the patient is on the table, place MRI-compatible electrodes (Quatrode from In Vivo) and ECG leads according to manufacturer's guidelines. Make sure that the ECG tracing shows in high (positive) $R$ waves. If it does not, either reposition the leads or toggle the lead polarity to alternative options, if available on the system.

Lead placement on the back is useful and may reduce motion artifacts related to breathing.

7. Place a pillow or other support under the knees to make the patient more comfortable.

8. Place a $22-\mathrm{G}$ intravenous catheter in the antecubital fossa if the gadolinium contrast is deemed necessary.

A power injector can be used to administer contrast, but is not necessary. 
9. Center the patient's chest in the phased-array coil.

10. Use the centering light to position the patient and put him or her into the center of the magnet.

Once this step has been performed, so long as the patient does not move on the table, the table itself can be moved and then replaced in the same position as before without jeopardizing the positioning of one scan relative to another.

11. If the patient is unable to hold still, provide an appropriate sedative.

12. Once the patient has been centered in the magnet, check again to be sure that the ECG tracing demonstrates sharp positive $\mathrm{R}$ waves for suitable triggering. If not, then with patient still positioned within the magnet, toggle lead polarity until a suitable tracing is obtained. If still not satisfactory, then bring the patient out of the magnet, check lead connections, and if necessary, reposition leads until a satisfactory tracing is obtained.

\section{Sequence 1: Rapid three-plane positioning scout}

13. For localization of subsequent acquisitions, run the system's three-plane scout scan (see Table A11.1.2). If possible, have the patient breath-hold at end expiration for the scout for better positioning of subsequent acquisitions.

Sequence 2: 2-D transverse double inversion recovery half-Fourier single-shot turbo spin echo (HASTE) (optional)

14. Use this optional ultrafast turbo spin-echo sequence (Table A11.1.3) for an overview of the chest and pericardium to localize lesions for subsequent spin-echo imaging. Position a series of transverse slices off the scout images to ensure coverage of the pericardium to the superior recess (to the level of the inferior aspect of the aortic arch). Instruct the patient to perform shallow breathing.

Table A11.1.2 Primary Clinical Imaging Parameters for Sequence 1 (Pilot Scan)

\begin{tabular}{ll}
\hline $\begin{array}{l}\text { Patient position } \\
\text { Scan type }\end{array}$ & $\begin{array}{l}\text { Supine } \\
\text { Gradient echo } \\
\text { Imaging plane (orientation) }\end{array}$ \\
$\begin{array}{l}\text { Three-plane (transverse, coronal, } \\
\text { sagittal) }\end{array}$ \\
Central slice or volume center & As short as possible \\
Echo time $\left(T_{\mathrm{E}}\right)$ & As short as possible \\
Repeat time $\left(T_{\mathrm{R}}\right)$ & $15^{\circ}$ \\
Flip angle $(\mathrm{FA})$ & $500 \mathrm{~mm}, 500 \mathrm{~mm}$ \\
Fields of view $\left(\mathrm{FOV}_{\mathrm{x}}, \mathrm{FOV}_{\mathrm{y}}\right)$ & $1.95 \mathrm{~mm}, 3.91 \mathrm{~mm}$ \\
Resolution $(\Delta x, \Delta y)$ & 256,128 \\
Number of data points collected $\left(N_{\mathrm{x}}, \mathrm{N}_{\mathrm{y}}\right)$ & 256,256 \\
Display matrix $\left(D_{\mathrm{x}}, \mathrm{D}_{\mathrm{y}}\right)$ & $10 \mathrm{~mm}$ \\
Slice thickness $(\Delta z)$ & $5-10$ \\
Number of slices & $0-10 \mathrm{~mm}$ \\
Slice gap & 1 \\
Number of acquisitions $\left(N_{\mathrm{acq}}\right)$ & No \\
Swap read and phase encoding & Variable \\
Slice location & Not applicable \\
Saturation pulses & $5-15 \mathrm{sec}$ \\
Scan time &
\end{tabular}


The sequence can be performed with breath-holding at end expiration, but this is not absolutely necessary. ECG gating is not necessary for this sequence but can be performed for better images.

\section{Sequence 3: 2-D transverse $T_{1}$-weighted turbo spin echo (TSE)}

15. Use the pilot scan and HASTE images to locate the pericardial mass in question or to define the extent of the pericardium.

For $T_{1}$-weighting, $T_{R}$ should be $\sim 85 \%$ to $90 \%$ of the patient's $R$-to-R interval (time between $R$ waves) and less than 900 msec. Short $T_{R}$ times will limit the number of slices that can be obtained in each acquisition. For greater coverage, this sequence can be run twice using the parameters shown in Table A11.1.4. If the area of coverage can be localized (e.g., for evaluation of a mass), the sequence need only be performed once with minimum slice gap. ECG gating is necessary in this sequence.

16. Acquisition times are too long to allow breath-hold imaging, and therefore with multiple acquisitions (signal averaging), instruct the patient to perform shallow breathing.

\section{Sequence 4: 2-D transverse $T_{2}$-weighted inversion-recovery turbo spin echo}

17. If necessary, perform limited transverse $T_{2}$-weighted turbo spin-echo images through the pericardium.

For example, using the HASTE and $T_{1}$-weighted TSE images, transverse $T_{2}$-weighted images can be performed through the mass in question, or through the pericardium at the level of an effusion, to help characterize the abnormality. An inversion-recovery technique will provide useful fat suppression and improve contrast between pathology and normal pericardial and epicardial fat. ECG gating is necessary in this sequence.

Table A11.1.3 Primary Clinical Imaging Parameters for Sequence 2 (HASTE, Optional)

\begin{tabular}{|c|c|}
\hline Patient position & Supine \\
\hline Scan type & Single-shot fast spin echo \\
\hline Imaging plane (orientation) & Transverse \\
\hline Central slice or volume center & Center of heart \\
\hline Echo time $\left(T_{\mathrm{E}}\right)$ & 43-63 msec (effective) \\
\hline Repeat time $\left(T_{\mathrm{R}}\right)$ & Infinity (echo spacing is $\sim 4 \mathrm{msec}$ ) \\
\hline Delay time $\left(T_{\mathrm{D}}\right)$ & $700 \mathrm{msec}$ \\
\hline Flip angle (FA) & $180^{\circ} a$ \\
\hline Fields of view $\left(\mathrm{FOV}_{\mathrm{x}}, \mathrm{FOV}_{\mathrm{y}}\right)$ & $\begin{array}{l}300-350 \mathrm{~mm}, 300 r-350 r \mathrm{~mm} \text {, } \\
\text { with } r=3 / 4 \text { (rectangular field of } \\
\text { view) depending on body habitus }\end{array}$ \\
\hline Resolution $(\Delta x, \Delta y)$ & $1.17-1.37 \mathrm{~mm}, 1.70-1.99 \mathrm{~mm}$ \\
\hline Number of data points collected $\left(N_{\mathrm{x}}, N_{\mathrm{y}}\right)$ & $\begin{array}{l}256,176 r, \text { with } r=3 / 4 \\
\text { (rectangular field of view) } \\
\text { depending on body habitus }\end{array}$ \\
\hline Display matrix $\left(D_{\mathrm{x}}, D_{\mathrm{y}}\right)$ & 256,256 \\
\hline Slice thickness $(\Delta z)$ & $5-8 \mathrm{~mm}$ \\
\hline Number of slices & 20 \\
\hline Slice gap & $0.8-2 \mathrm{~mm}$ \\
\hline Number of acquisitions $\left(N_{\mathrm{acq}}\right)$ & 1 \\
\hline Swap read and phase encoding & No \\
\hline Saturation pulses & No \\
\hline ECG gating & Not necessary \\
\hline Scan time & $20-30 \mathrm{sec}$ \\
\hline
\end{tabular}


18. Set the imaging parameters as shown in Table A11.1.5 to obtain a single slice per breath-hold. Ensure that the $T_{\mathrm{R}}$ plus delay time is less than $85 \%$ to $90 \%$ of two R-to-R intervals. Instruct the patient to breath-hold at end expiration, if possible, for best reproducibility.

19. Determine if the inversion time needs to be adjusted.

The inversion time determines the degree of fat suppression. Delay times are selected to allow imaging in diastole.

\section{Sequence 5: 2-D transverse and coronal fast cine gradient echo}

20. For global pericardial disease, such as pericarditis, perform transverse fast cine gradient-echo images through the entire pericardium, acquiring additional selected sagittal images for evaluation of the inferior pericardium.

Table A11.1.4 Primary Clinical Imaging Parameters for Sequence 3 ( $T_{1}$-Weighted TSE)

\begin{tabular}{|c|c|}
\hline Patient position & Supine \\
\hline Scan type & Fast spin echo \\
\hline Imaging plane (orientation) & Transverse \\
\hline Central slice or volume center & Center of heart \\
\hline Echo time $\left(T_{\mathrm{E}}\right)$ & $12 \mathrm{msec}$ (or minimum) \\
\hline Ech train length (ETL) & 3 \\
\hline Repeat time $\left(T_{\mathrm{R}}\right)$ & $\begin{array}{l}<900 \text { msec (and }<90 \% \text { of the } \\
\text { R-to-R interval) }\end{array}$ \\
\hline Delay time after $\mathrm{R}$ wave & $0 \mathrm{msec}$ \\
\hline Flip angle (FA) & $180^{\circ} a$ \\
\hline Fields of view $\left(\mathrm{FOV}_{x}, \mathrm{FOV}_{y}\right)$ & $\begin{array}{l}300-350 \mathrm{~mm}, 300 r-350 r \mathrm{~mm}, \\
\text { with } r=3 / 4 \text { (rectangular field of } \\
\text { view) depending on body habitus }\end{array}$ \\
\hline Resolution $(\Delta x, \Delta y)$ & $1.17-1.37 \mathrm{~mm}, 1.43-1.67 \mathrm{~mm}$ \\
\hline Number of data points collected $\left(N_{x}, N_{y}\right)$ & $\begin{array}{l}256,210 r, \text { with } r=3 / 4 \\
\text { (rectangular field of view) } \\
\text { depending on body habitus }\end{array}$ \\
\hline Display matrix $\left(D_{x}, D_{y}\right)$ & 256,256 \\
\hline Slice thickness $(\Delta z)$ & $4-8 \mathrm{~mm}$ \\
\hline Number of slices & $10-12$ \\
\hline Slice gap & $\begin{array}{l}0.4-0.8 \mathrm{~mm}^{\text {or }} 4-8 \mathrm{~mm} \text { if } \\
\text { concatenated }^{b}\end{array}$ \\
\hline Number of acquisitions $\left(N_{\text {acq }}\right)$ & $3-5$ \\
\hline Swap read and phase encoding & No \\
\hline Saturation pulses & No \\
\hline ECG gating & Yes \\
\hline Scan time & $3-8 \mathrm{~min}$ \\
\hline \multicolumn{2}{|c|}{$\begin{array}{l}{ }^{a} \text { The system displays the flip angle of the refocusing pulse. The flip angle of the first pulse of this } \\
\text { sequence is } 90^{\circ} \text {. } \\
{ }^{b} \text { If the } z \text {-axis coverage needed is on the order of } 60 \text { to } 80 \mathrm{~mm} \text {, then a single acquisition with about } 8 \\
\text { to } 10 \text { slices at } 6 \text { to } 8 \mathrm{~mm} \text { thickness and minimal gap }(0.6 \text { to } 0.8 \mathrm{~mm} \text { ) can be performed. For complete } \\
\text { coverage of the chest, two separate acquisitions of } 8 \text { to } 10 \text { slices can be performed, each with a gap } \\
\text { equal to the slice thickness (e.g., } 8 \mathrm{~mm} \text { slice thickness with } 8 \mathrm{~mm} \text { gap). In this example, the second } \\
\text { acquisition should be performed with the slice positions shifted by } 8 \mathrm{~mm} \text { and with the same thickness } \\
\text { and gap, so that with the two acquisitions together, complete coverage of the chest will be obtained } \\
\text { with } 8 \text {-mm-thick slices and effectively no gap. }\end{array}$} \\
\hline
\end{tabular}


Table A11.1.5 Primary Clinical Imaging Parameters for Sequence 4 (Short Tau Inversion Recovery)

\begin{tabular}{ll}
\hline Patient position & Supine \\
Scan type & Inversion recovery fast spin echo \\
Imaging plane (orientation) & Transverse \\
Central slice or volume center & Center of heart \\
Echo time $\left(T_{\mathrm{E}}\right)$ & $57-76 \mathrm{msec}$ \\
Echo train length (ETL) & 33 \\
Repeat time $\left(T_{\mathrm{R}}\right)$ & $<900 \mathrm{msec}\left(T_{\mathrm{R}}+T_{\mathrm{D}}\right.$ should be $<2$ \\
& $\times(85 \%-90 \%$ of the R-to-R \\
& interval)) \\
Inversion time $\left(T_{\mathrm{I}}\right)$ & $170 \mathrm{msec}$ \\
Delay time $\left(T_{\mathrm{D}}\right)$ & $500 \mathrm{msec}$ \\
Flip angle $(\mathrm{FA})$ & $180^{\circ}$ \\
Fields of view $\left(\mathrm{FOV}, \mathrm{FOV}_{y}\right)$ & $300-350 \mathrm{~mm}, 300 r-350 r$ mm, \\
& with $r=3 / 4($ rectangular field of \\
& view) depending on body habitus \\
Resolution $(\Delta x, \Delta y)$ & $1.17-1.37$ mm, $1.67-1.94$ mm \\
Number of data points collected $\left(N_{x}, N_{y}\right)$ & $256,180 r$, with $r=3 / 4$ \\
& $($ rectangular field of view) \\
Display matrix $\left(D_{x}, D_{y}\right)$ & depending on body habitus \\
Slice thickness $(\Delta z)$ & 256,256 \\
Number of slices & 8 mm \\
Slice gap & 1 \\
Number of acquisitions $\left(N_{\text {acq }}\right)$ & Not applicable \\
Swap read and phase encoding & 1 \\
Saturation pulses & No \\
ECG gating & No \\
Scan time & Yes \\
\hline & $12-15$ sec \\
\hline &
\end{tabular}

Cine gradient echo images can be performed selectively. For evaluation of the effect of pericardial masses on hemodynamics, acquisitions can be positioned selectively. ECG gating is necessary in this sequence.

21. Set the imaging parameters as shown in Table A11.1.6 to obtain a single slice per breath-hold (parameters shown are for a segmented $k$ space approach with nine lines of $k$ space per segment and four echoes shared). Adjust the parameters depending on the patient's breath-holding capability and heart rate. For patients with slower heart rates, use sequences that provide a greater number of lines per segment to help reduce acquisition times. Set the number of phases of the cardiac study according to the formula (for view sharing off):

Number of cardiac phases $=(\mathrm{R}$-to-R interval $) \times 85 \% / T_{\mathrm{R}}$

22. Instruct the patient to breath-hold at end expiration, if possible, for best reproducibility. If patient is unable to breath-hold, perform the sequence with multiple acquisitions (e.g., three signal averages) with the patient breathing normally or shallowly. 
Table A11.1.6 Primary Clinical Imaging Parameters for Sequence 5 (Cine Gradient Echo)

\begin{tabular}{|c|c|}
\hline Patient position & Supine \\
\hline Scan type & $\begin{array}{l}\text { Segmented } k \text {-space cine gradient } \\
\text { echo }\end{array}$ \\
\hline Imaging plane (orientation) & Transverse (coronal is optional) \\
\hline Central slice or volume center & Center of heart \\
\hline Echo time $\left(T_{\mathrm{E}}\right)$ & $4.8 \mathrm{msec}$ (or minimum) \\
\hline Number of lines per segment & 9 (see step 21$)$ \\
\hline Repeat time $\left(T_{\mathrm{R}}\right)$ & $80-100 \mathrm{msec}$ (temporal resolution) \\
\hline Delay time $\left(T_{\mathrm{D}}\right)$ & $0 \mathrm{msec}$ \\
\hline Flip angle (FA) & $20^{\circ}$ \\
\hline Fields of view $\left(\mathrm{FOV}_{x}, \mathrm{FOV}_{y}\right)$ & $\begin{array}{l}300-350 \mathrm{~mm}, 300 r-350 r \mathrm{~mm} \text {, } \\
\text { with } r=3 / 4 \text { (rectangular field of } \\
\text { view) depending on body habitus }\end{array}$ \\
\hline Resolution $(\Delta x, \Delta y)$ & $1.17-1.37 \mathrm{~mm}, 1.70-1.99 \mathrm{~mm}$ \\
\hline Number of data points collected $\left(N_{x}, N_{y}\right)$ & $\begin{array}{l}256,176 r, \text { with } r=3 / 4 \\
\text { (rectangular field of view) } \\
\text { depending on body habitus }\end{array}$ \\
\hline Display matrix $\left(D_{x}, D_{y}\right)$ & 256,256 \\
\hline Slice thickness $(\Delta z)$ & $5-7 \mathrm{~mm}$ \\
\hline Number of slices & 1 \\
\hline Slice gap & Not applicable \\
\hline Number of acquisitions $\left(N_{\text {acq }}\right)$ & 1 \\
\hline Swap read and phase encoding & No \\
\hline Saturation pulses & No \\
\hline Number of cardiac phases & $($ R-to- $\mathrm{R}$ interval $) \times 85 \% / T_{\mathrm{R}}$ \\
\hline ECG gating & Yes \\
\hline Scan time & $15-20 \mathrm{sec}$ \\
\hline
\end{tabular}

\section{IMAGING OF NEOPLASTIC PERICARDIUM}

For patients with suspected neoplastic involvement of the pericardium, contrast-enhanced images may also be needed in addition to the Basic Protocol. Intravenous gadolinium contrast (typically, $0.1 \mathrm{mmol} / \mathrm{kg}$ ) is administered, and turbo $T_{1}$ spin-echo images are obtained. If necessary, nonenhanced images can be subtracted from contrast-enhanced images using postprocessing software to depict enhancing lesions.

\section{Materials}

Normal saline $(0.9 \% \mathrm{NaCl})$, sterile

Gadolinium-based MR contrast agent (e.g., Magnevist, Omniscan, or Prohance)

\section{Set up equipment and patient}

1. Use the same equipment and perform equipment and patient setup as for the previous method (see Basic Protocol).

\section{Run sequences 1 to 5 .}

3. Leaving the patient in the magnet, inject the contrast agent, and flush the line with $20 \mathrm{ml}$ saline.

A dose of $0.1 \mathrm{mmol} / \mathrm{kg}$ of contrast agent is usually given.

Acquired Heart

Disease

A11.1.7 
Sequence 6: 2-D Transverse $T_{1}$-weighted turbo spin echo

4. Repeat the sequence described in Table A11.1.4 3 to 5 min after intravenous injection of gadolinium contrast material. Ensure that all imaging parameters are kept the same to facilitate postprocessing.

\section{COMMENTARY}

\section{Background Information}

The pericardium is a fibroserous sac that contains the heart and proximal portions of the ascending aorta and main pulmonary artery. By MRI, it appears as a thin low-signal-intensity line that should measure no greater than $4 \mathrm{~mm}$ in thickness (Fig. A11.1.1). It is typically well delineated by MRI because it is surrounded by epicardial and mediastinal fat. There is normally a small amount of fluid, typically 15 to $50 \mathrm{ml}$, that lubricates the pericardial sac.

Transthoracic echocardiography is considered the first-line diagnostic modality for patients with suspected pericardial disease (Martin et al., 1998). However, pericardial thickening in the absence of pericardial fluid is difficult to diagnose, such as in the setting of constrictive pericarditis. Certain patients have poor acoustic windows, and the imaging technique is extremely dependent on the skill of the echocardiographer. Computed tomography (CT) imaging offers wide field-of-view imaging. Its sensitivity for the detection of pericardial calcifications, as seen in constrictive pericarditis, is advantageous. However, tissue contrast is otherwise inferior to MRI, and motion artifacts limit visualization of the noncalcified pericardium. However, with the advent of multidetector ECG-gated CT, dynamic acquisitions may become possible.

Indications for MRI of the pericardium include the diagnosis of constrictive pericarditis, evaluation of pericardial effusions, including differentiation of hemorrhagic from nonhemorrhagic effusions, detection of primary or secondary pericardial neoplastic disease, and evaluation of congenital anomalies of the pericardium.

The distinction between constrictive pericarditis and restrictive cardiomyopathy can be a difficult clinical problem since clinical presentation and hemodynamic patterns are similar (Masui et al., 1992). Although unable to visualize calcifications, MRI is still an ideal technique for diagnosing constrictive pericarditis. Typical findings include pericardial thickening $>4 \mathrm{~mm}$, dilated inferior vena cava, dilated atria, and compressed ventricles (Fig. A11.1.2).
Thickening can be confined to the right side of the heart, and abnormal motion of the interventricular septum may be seen during early diastole. Patients with restrictive cardiomyopathy do not usually have thickened pericardium.

Pericardial effusions can develop from diseases such as uremia, collagen vascular diseases, postpericardiotomy, and postinfarction syndromes, as well as viral, tuberculous, or idiopathic acute pericarditis and neoplasia. Magnetic resonance imaging can be used to help characterize fluid collections and to detect the presence of adhesions. Transudative effusions are typically low in signal intensity on $T_{1}$-weighted images and high on $T_{2}$-weighted images. Proteinaceous or hemorrhagic effusions may be high in signal intensity on $T_{1^{-}}$ weighted images.

The most common pericardial mass is a pericardial cyst, which classically lies along the right cardiophrenic angle and demonstrates uniform high signal intensity on $T_{2}$-weighted images (Fig. A11.1.3). Neoplastic involvement is more commonly due to secondary involvement such as from breast and lung carcinoma, lymphoma, and melanoma. When direct extension of tumor occurs, the pericardial effusion is typically hemorrhagic. Mesothelioma is the most common malignant pericardial tumor (Hancock, 1990). In cases of suspected neoplastic disease, contrast-enhanced imaging may be necessary to identify and delineate tumor that is associated with complex pericardial effusions. MRI can also help to differentiate pericardial disease from adjacent paracardiac pathology (Barakos et al., 1989; Fig. A11.1.4).

Congenital absence of the pericardium, typically partial and left sided, can be evident by chest radiography and is readily confirmed by unenhanced MRI.

\section{Critical Parameters and Troubleshooting}

\section{ECG tracings}

Since all but the HASTE sequences used for imaging the pericardium must be ECG gated,
Pericardial Disease

A11.1.8 


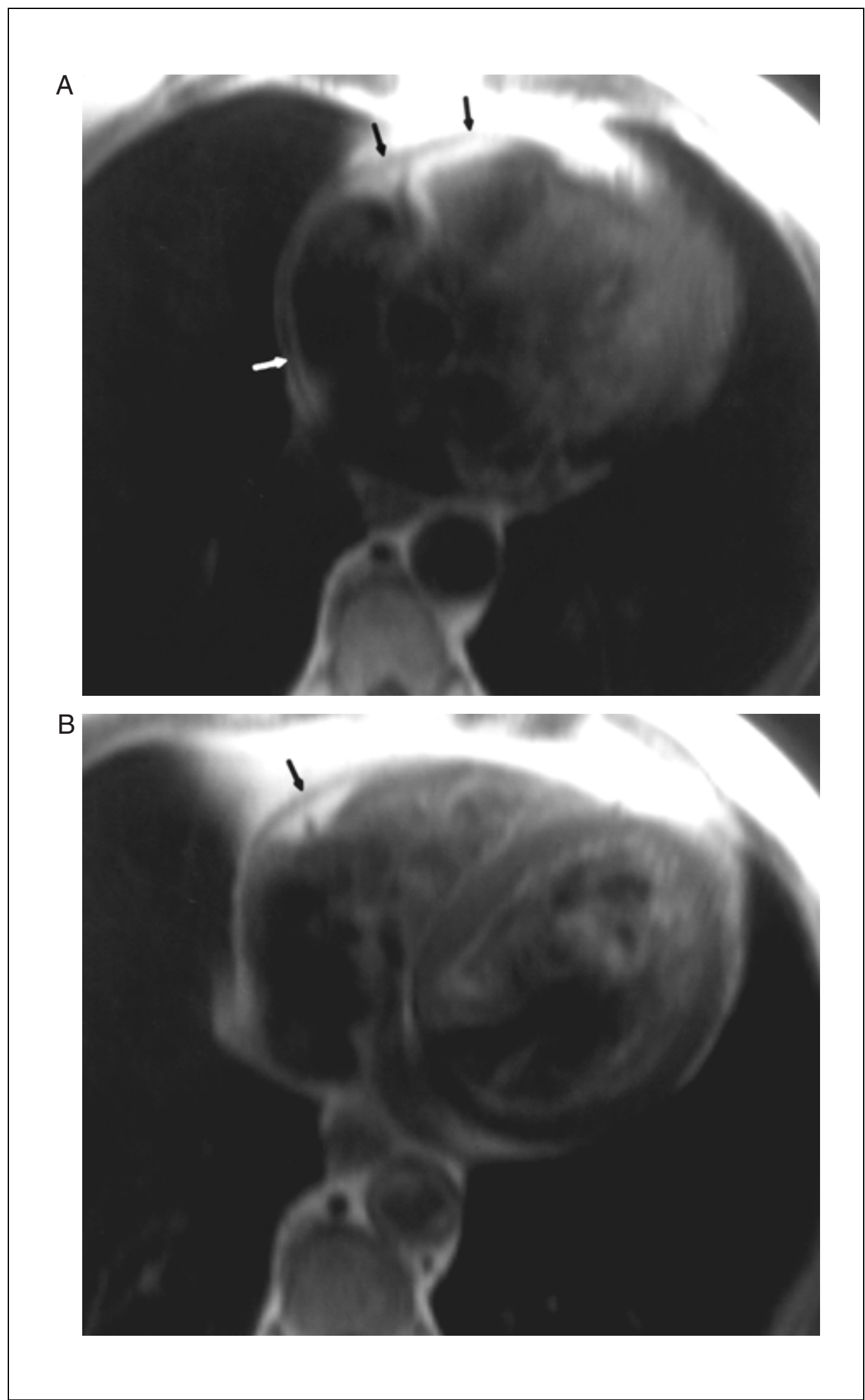

Figure A11.1.1 Normal pericardium. (A, B) Transverse HASTE images at two levels demonstrating normal low signal intensity line measuring $<4 \mathrm{~mm}$, corresponding to normal pericardium (arrows). 
A

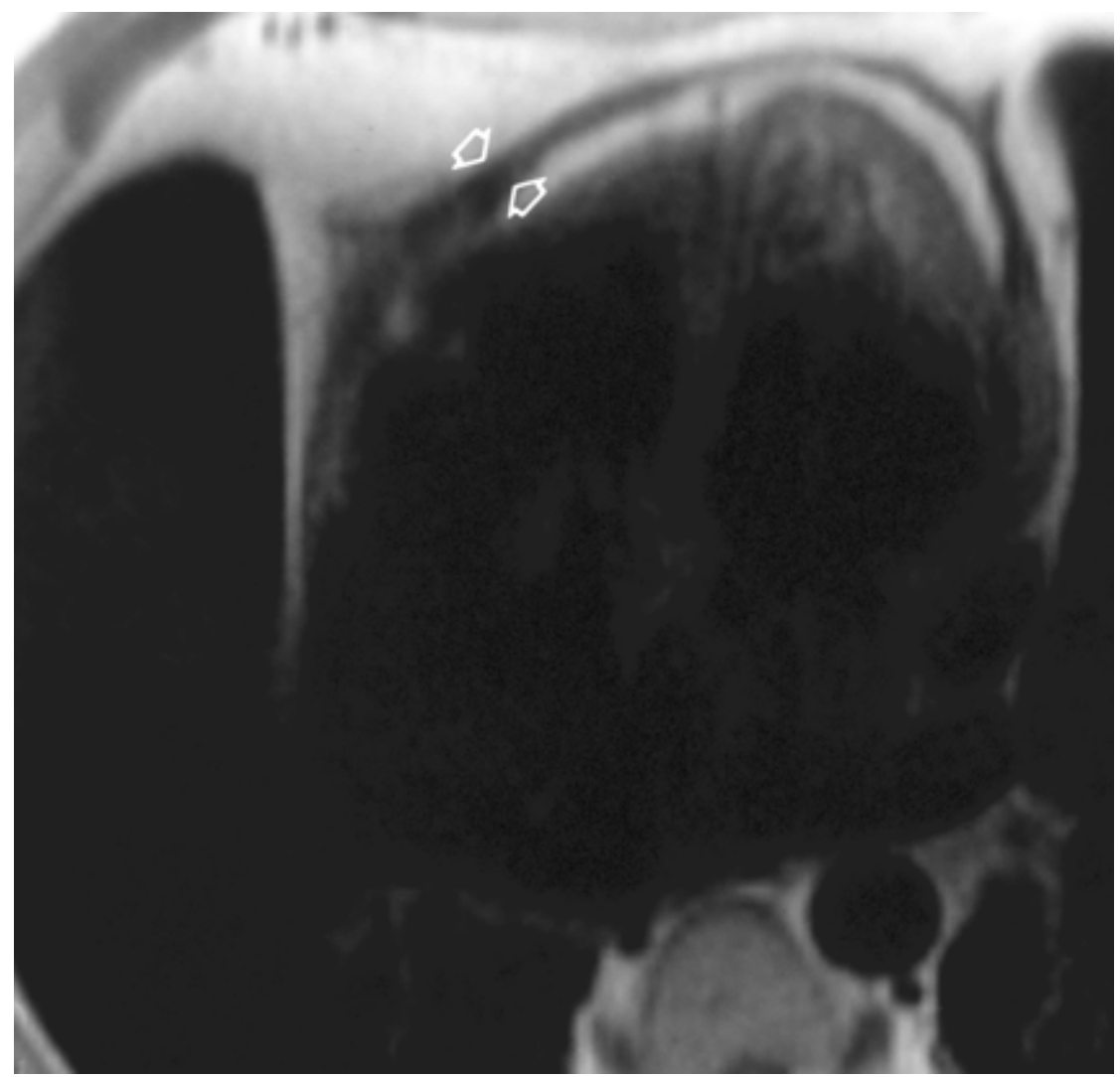

B

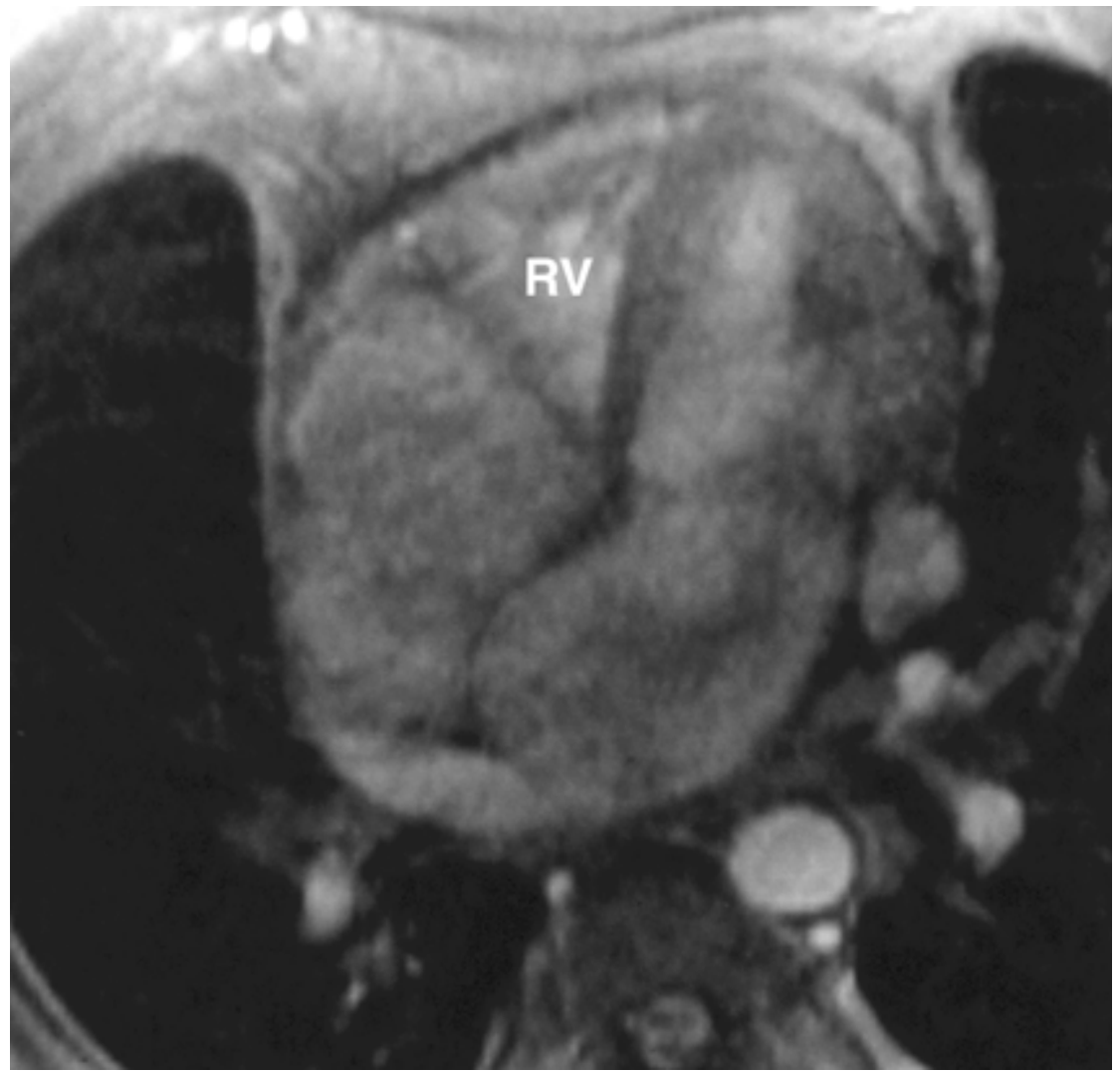

Pericardial Disease

A11.1.10
Figure A11.1.2 Constrictive pericarditis. (A) Transverse HASTE and (B) transverse cine gradientecho image show thickening of the pericardium (arrowheads), enlargement of the atria, and associated compression of the right ventricle (RV). 


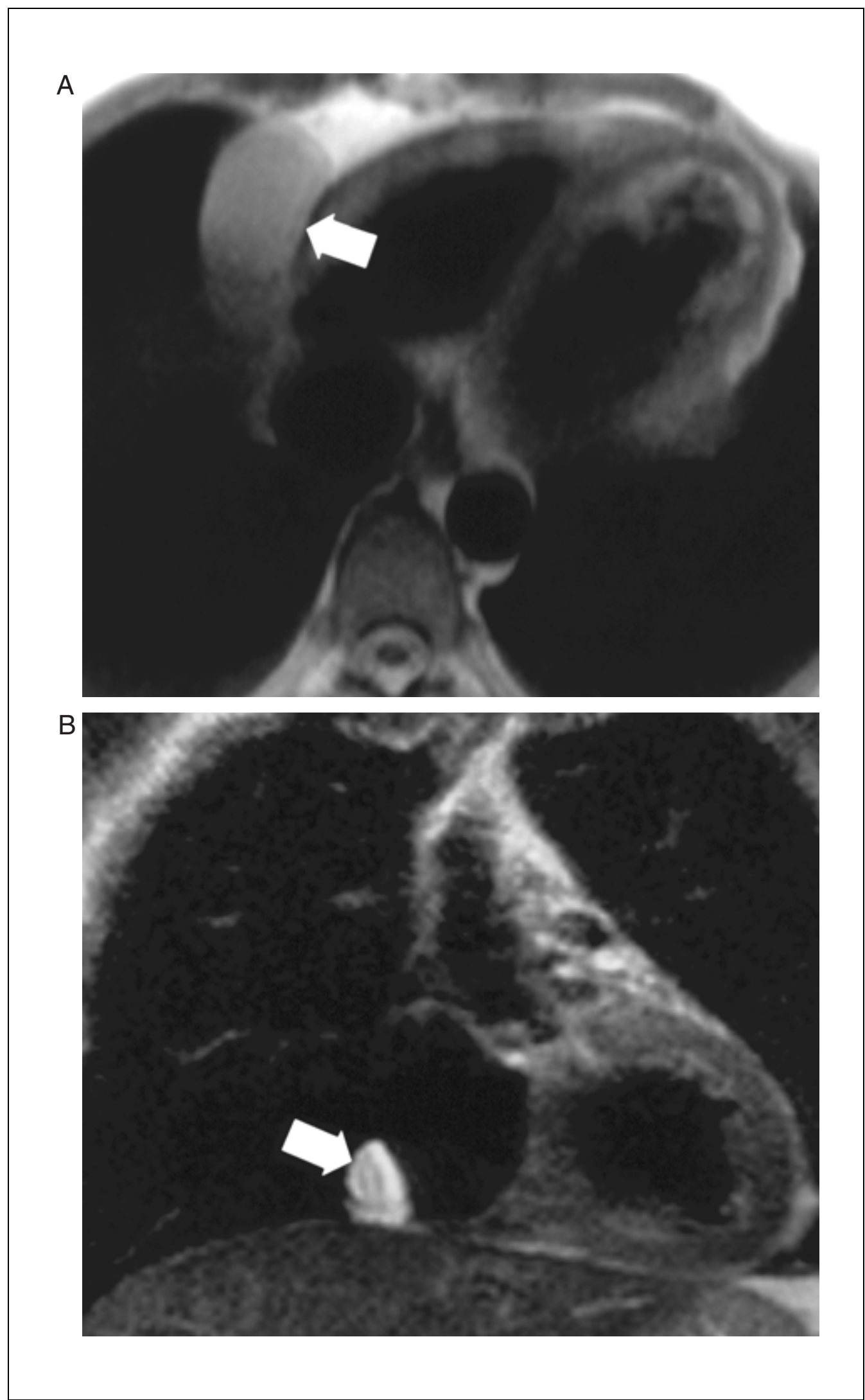

Figure A11.1.3 Pericardial cyst. (A) Transverse and (B) coronal HASTE images show a well-defined oval mass (arrows) with high signal intensity on $T_{2}$-weighted images in the right cardiophrenic angle, a characteristic location for a pericardial cyst. Contrast-enhanced images (not shown) confirmed a lack of enhancement in this lesion. 
A

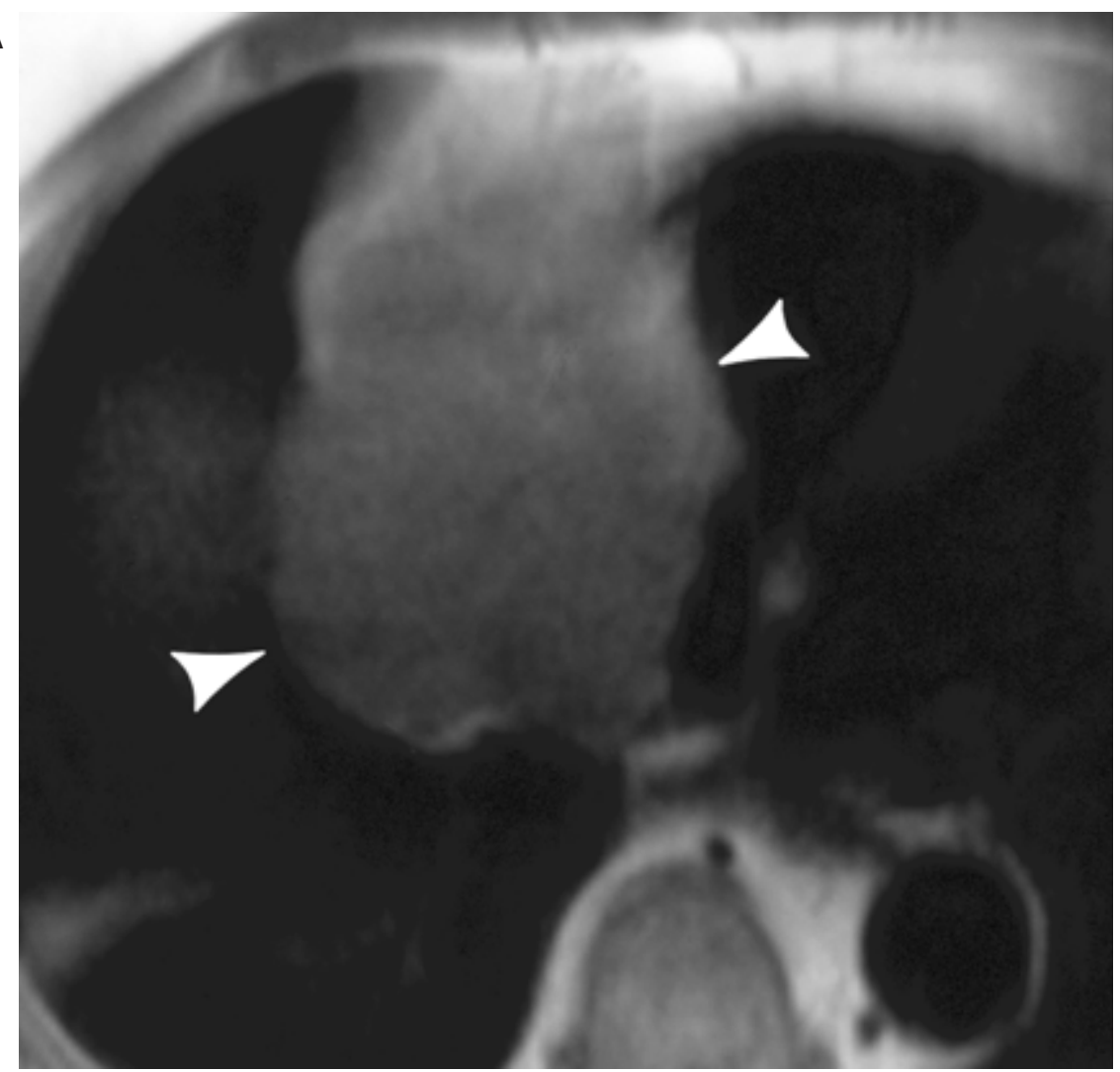

B

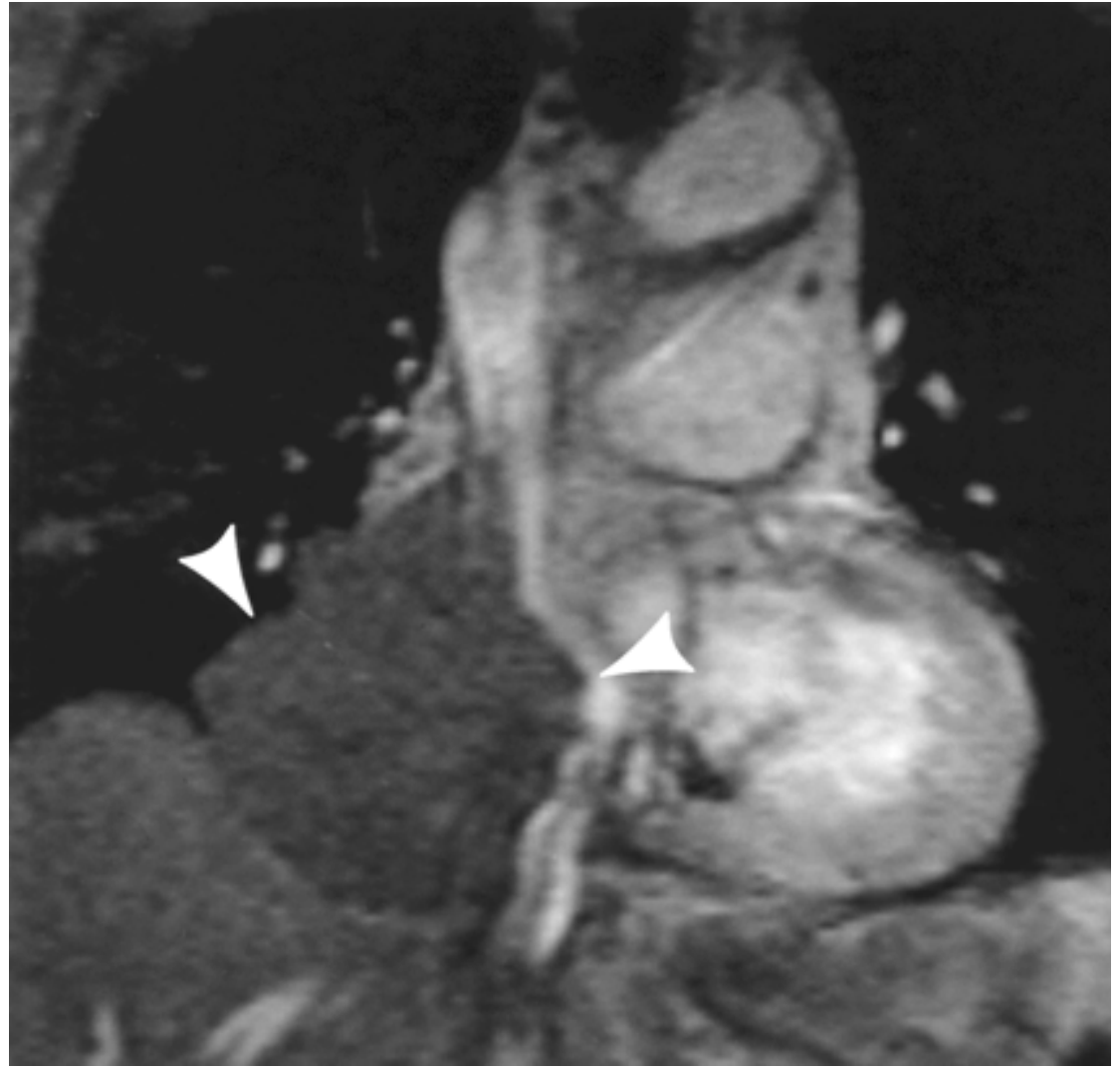

Pericardial Disease

A11.1.12
Figure A11.1.4 Pericardial and cardiac lymphoma. (A) Transverse HASTE image and (B) coronal cine gradient-echo image of the heart demonstrate a large mass (arrows) that invades through the pericardial space into the right heart in a patient with lymphoma. 
it is vital that an adequate ECG tracing be obtained while the patient is in the bore of the magnet. For an ideal ECG tracing, sharp up-going $\mathrm{R}$ waves with significantly smaller (or inverted) $\mathrm{T}$ waves are needed, as triggering typically depends on the ECG value crossing a particular threshold. Magnetic resonancecompatible ECG leads with conductive gel should be used. For good skin contact, the patient's skin may need to be shaved in some cases. The position of the heart and axes of the conduction pathways may vary considerably across patients. The manufacturer's recommendations for lead positioning should be used as a starting point. If polarity is not as desired (e.g., inverted $\mathrm{R}$ waves), check that leads are positioned correctly (RA to the right side, LA to the left side, etc.), and then if needed, toggle through the lead polarity options (I, II, AVR, etc.). Finally, as a last resort, reposition ECG leads, using new adhesive pads to ensure adequate adhesion and conduction gel. If ECG tracings are lost during positioning of the patient or scanning, check the patient to ensure that leads have remained properly connected.

\section{Customization of gated MRI parameters}

Magnetic resonance imaging of the heart and pericardium requires careful customization of imaging parameters according to patient heart rate and breath-holding capability. For a multislice turbo spin-echo sequence, the number of slices that can be imaged in one acquisition depends on $T_{\mathrm{R}}$, which in turn depends on the patient's heart rate. The $T_{\mathrm{R}}$ must be defined as shorter than the R-to- $\mathrm{R}$ interval (which is inversely related to patient heart rate) to ensure an adequate trigger window. Typically, trigger windows of $10 \%$ to $15 \%$ are needed to allow for variability of heart rates. For patients who have extremely variable heart rates (e.g., frequent premature beats), then the shorter R-to-R intervals should be used to set $T_{\mathrm{R}}$

For single-slice cine gradient-echo images, multiple phases of the heart cycle are imaged at a single slice position. The lines of $k$ space (number of phase-encoding lines) collected for each cardiac phase during each R-to-R interval defines the number of lines per segment. The more lines per segment, the longer the $T_{\mathrm{R}}$ and the fewer the cardiac phases that can be acquired (the $T_{\mathrm{R}}$ times the number of cardiac phases should equal $\sim 90 \%$ of the R-to-R interval). The total number of heartbeats needed to acquire sufficient data to generate all images depends on the total number of phase encoding steps needed and the number of lines per segment (number of heart beats should equal approximately the number of phase encoding lines per image divided by the number of lines per segment). While sequences could be customized for every patient, to simplify the process, system protocols can be set up for specific categories of patients, such as "slow heart rate" and "fast heart rate," with the aim of limiting acquisition times to $<20 \mathrm{sec}$.

On some systems, a view-sharing option will be available for cine gradient-echo sequences that enables almost double or greater temporal resolution (number of cardiac phases) in the same acquisition time.

\section{Motion or breathing artifacts}

Occasionally, despite a patient's effort to breath-hold, substantial motion artifact is seen on the MRI. Cine gradient-echo images can be performed with multiple signal averages with the patient breathing normally but shallowly. An anterior saturation band can be placed over the anterior chest wall subcutaneous fat to help reduce motion-related artifacts from breathing.

\section{Anticipated Results}

The goals of MRI of the pericardium are to visualize the pericardium, characterize abnormalities of the pericardium itself or the pericardial space, and identify and characterize pericardial masses. With ECG gating, MRI can provide clear visualization of anatomic abnormalities, such as pericardial thickening or pericardial effusions, as well as allow an assessment of consequent hemodynamic abnormalities. For example, in patients with constrictive pericarditis, the effect of constriction on the right ventricle can be seen during dynamic cine gradient-echo imaging. Similarly, in patients with large pericardial effusions, cine gradientecho images depict the compression of rightsided heart chambers, which indicates physiologically significant changes of tamponade.

The distinction between pericardial disease and paracardial masses can also be performed using MRI. With pericardial disease, involvement of the pericardium can be visualized directly. The use of contrast material can supplement cine gradient-echo imaging in helping to depict the extent of enhancing pericardial neoplasm in the setting of a complex pericardial effusion. However, in many cases, contrast material is not needed to diagnose and characterize pericardial pathology.
Acquired Heart

Disease

A11.1.13 


\section{Literature Cited}

Barakos, J.A., Brown, J.J., and Higgins, C.B. 1989. MR imaging of secondary cardiac and paracardiac lesions. Am. J. Roentgenol. 153:47-50.

Hancock, E.W. 1990. Neoplastic pericardial disease. Cardiol. Clin. 8:673-682.

Martin, E.T., Fuisz, A.R., and Pohost, G.M. 1998 Imaging cardiac structure and pump function. Cardiol. Clin. 16:135-160

Masui, T., Finck, S., and Higgins, C.B. 1992. Constrictive pericarditis and restrictive cardiomyopathy: Evaluation with MR imaging. Radiology 182:369-373.

\section{Key References}

Dupuis, K., Thangaraj, V., and Edelman, R.R. 1996. Practical MRI for the technologist and imaging specialist. In Clinical Magnetic Resonance Imaging (R.R. Edelman, J.R. Hesselink, and M.B. Zlatkin, eds.) pp. 52-87. W.B. Saunders, Philadelphia.
Covers a wide range of practical information for setting patients up for MRI and also includes a useful description of cardiac imaging sequence parameters.

Higgins, C.B. 1992. Essentials of Cardiac Radiology and Imaging. J.B. Lippincott, New York.

Contains a detailed description of the spectrum of modalities that can be used to image the pericardium, including plain film radiography and MRI.

White, C.S. 1996. MR evaluation of the pericardium and cardiac malignancies. MRI Clin. North Am. 4:237-251.

Reviews the anatomy and pathology of the pericardium with an emphasis on MRI findings.

Contributed by Vivian S. Lee

New York University Medical Center

New York, New York 